\title{
Estimation of soil pore-water pressure variations using a thin plate spline basis function
}

\author{
M. R. Mustafa ${ }^{1}$, R. B. Rezaur ${ }^{2}$, M. H. Isa ${ }^{1} \&$ H. Rahardjo ${ }^{3}$ \\ ${ }^{1}$ Department of Civil Engineering, \\ Universiti Teknologi PETRONAS, Malaysia \\ ${ }^{2}$ Golder Associates Ltd. Calgary, Canada \\ ${ }^{3}$ School of Civil and Environmental Engineering, \\ Nanyang Technological University, Singapore
}

\begin{abstract}
Information of soil pore-water pressure changes due to climatic effect is an integral part for studies associated with hill slope analysis. Soil pore-water pressure variations in a soil slope due to rainfall were predicted using Artificial Neural Network (ANN) technique with Thin Plate Spline (TPS) radial basis function. A radial basis function (RBF) neural network with network architecture of 8-36-1 (input-hidden-output) was selected to develop RBF model. Number of hidden neurons was selected using trial and error procedure whereas spread of the basis function was established using normalization method. Time series data of rainfall and pore-water pressure was used for training and testing the RBF model. The performance of the model was evaluated using root mean square error, coefficient of correlation and coefficient of efficiency. The results of the model prediction revealed that the model produced promising results indicating that TPS basis function is able to predict time series of pore-water pressure responses to rainfall. Comparison with other studies showed that the RBF model using TPS basis function can be used as alternate of Gaussian basis function for prediction of soil pore-water pressure variations.
\end{abstract}

Keywords: neural network, pore-water pressure, prediction, thin plate spline, radial basis function, rainfall. 


\section{Introduction}

Soil pore-water pressure reveals a highly nonlinear and complex behavior due to climatic changes in tropical regions particularly because of the spatial and temporal variability of rainfall, temperature, evaporation and soil characteristics. Excessive increase or highly positive pore-water pressure may lead to slope failures in land slide susceptible areas. Therefore, the knowledge of pore-water pressure is highly desirable in every slope stability analysis and studies related to hill slopes. In general, soil pore-water pressure variations highly depends on several factors i.e. rainfall, soil properties (grain size, porosity, density etc.), temperature, evaporation, solar radiation, soil depth, and antecedent conditions. Thus, reliable prediction of pore-water pressure is considerably data-demanding. However, few studies on prediction of soil pore-water pressure variations using artificial neural network techniques have been observed in recent years [1-3].

Applications of artificial neural network (ANN) techniques to solve complex problems in civil engineering initiated in the late 1980s [4]. The extensive range of ANN applications for simulation, prediction and forecasting different variables in water resources engineering has been observed in recent years [1-3, 5-11]. However, it appears that use of ANN applications to predict soil porewater pressure is relatively few [1-3]. Mustafa et al. [2] predicted soil pore-water pressure using scaled conjugate training algorithm in multilayer perceptron neural network. They obtained promising results and stated that neural network using scaled conjugate training algorithm is appropriate to estimate the porewater pressures. Mustafa et al. [1] estimated the pore-water pressure using radial basis function neural network technique. They used Gaussian basis function to predict the pore-water pressure at two different sites with the same model. They investigated that the model trained with a dataset of slope but it was tested using the dataset of different slope having similar soil characteristics. Furthermore, they investigated that complex and nonlinear behavior of soil pore-water pressure although a function of a number of variables but can be predicted using RBF modeling with a modest number of input variables.

Since, few studies have been observed to predict soil pore-water pressure using ANN. However, it appears radial basis function neural network using thin plate spline basis function has, so far, not been used to prediction soil pore-water pressure variations in response to rainfall. Therefore, the objective of this study is to predict soil pore-water pressure variations in response to rainfall using thin plate spline basis function.

\section{Data source and study area}

The time series of rainfall data and pore-water pressures recorded at soil depth of $0.5 \mathrm{~m}$ for a period of 13 months were used. The data were collected in 19981999 through a field instrumentation program of a residual soil slope in Yishun, Singapore [12]. The maximum and minimum values of pore-water pressure in the data are $5.5 \mathrm{kPa}$ and $-55.9 \mathrm{KPa}$ respectively. The entire monitoring for porewater pressure measurements were obtained at four different soil depths (i.e. 
depth of $0.5,1,2$, and $3 \mathrm{~m}$ ). The primarily objective of data collection was to understand rainfall-induced slope failure mechanism and behavior of hydrological responses to the slopes in tropical climate conditions. Climate at the study area is hot and humid equatorial with no marked dry season. The temperature variations at the site are very few throughout the year with an annual average temperature of $26.6^{\circ} \mathrm{C}$ and a relative humidity of $84 \%$ [13]. Generally the soil found at the study area consist of residual soils from the Bukit Timah granite with small variation from silty or clayey sands to silty or sandy clays, subjected to the degree of weathering, but are commonly sandy clayey silt [12].

\section{Radial Basis Function neural network}

Radial Basis Function neural network was recognized as an alternative to Multilayer feed forward neural network in the late 1980s. The derivation of RBF is established through classical techniques like clustering, functional approximation, spline interpolation and mixture models [14]. Considering the complex and highly non-linear behavior of rainfall and pore-water pressure, the most appropriate neural network for modeling rainfall pore-water pressure behavior should have the capability to capture any continuous function; like radial basis function. Previous studies on application of RBF type ANN to water resources engineering problems indicate that $\mathrm{RBF}$ neural network presents superior generalization capability to capture complex natural non-linear behavior.

\subsection{RBF model architecture}

The RBF neural network model developed consists of three layers. Hereafter will be called as RBF-TPS model; RBF-TPS stands for Radial Basis Function using Thin Plate Spline function. The first layer is known as input layer, the second layer is called hidden or radial basis function layer and the third layer is known as output or linear layer. Radial basis function or hidden neurons in the hidden layer are denoted by HN (Figure 1). The selection of appropriate architecture particularly the number of input neurons is highly important to achieve an effective and efficient model. Recently, Mustafa et al. [1] investigated that the number of input neurons must contain the antecedent values of rainfall and porewater pressure while establishing a neural network model for estimation of porewater pressure variation. They examined the rainfall and pore-water pressure data from autocorrelation analysis within the dataset and cross-correlation analysis between both datasets (i.e. rainfall and pore-water pressure data). They observed that current pore-water pressure value is highly dependent on the five antecedent pore-water pressure values. Furthermore, two antecedent values in rainfall data have greater influence on the current value of the pore-water pressure. Therefore, they suggested that eight numbers of inputs containing five antecedent pore-water pressures, two antecedent rainfall values and one current rainfall would be appropriate to predict current pore-water pressure. Therefore, in this study, a similar configuration was adopted for the number of neurons in 
the input layer. Thus, the input neurons structure is represented by the following equation.

$$
\mathrm{P}_{\mathrm{t}}=f\left(\mathrm{R}_{\mathrm{t}}, \mathrm{R}_{\mathrm{t}-1}, \mathrm{R}_{\mathrm{t}-2}, \mathrm{P}_{\mathrm{t}-1}, \mathrm{P}_{\mathrm{t}-2}, \mathrm{P}_{\mathrm{t}-3}, \mathrm{P}_{\mathrm{t}-4}, \mathrm{P}_{\mathrm{t}-5}\right)
$$

where, $\mathrm{P}$ and $\mathrm{R}$ represent the pore-water pressure and rainfall values. Whereas, $\mathrm{t}$ is current time and $t-1$ to $t-5$ are the one to five steps antecedent time data. There is no hard and fast rule to select the number of basis functions or hidden neurons in the hidden layer. Therefore, in this study the appropriate number of hidden neurons was selected by trial and error. It was observed that 36 numbers of hidden neurons were sufficient to adopt the nonlinear pattern between the inputs and outputs variables. Since, the output expected from the network is only one (current pore-water pressure). Therefore, the output layer consists of one neuron only. Thus the structure of the selected network can be written as 8-36-1 (i.e. input-hidden-output neurons). The spread of the basis function was selected using normalization method of spread determination. According to this method the spread is twice the average difference between the successive centers of the basis functions [15]. The value of spread for the basis function is 1.8.

\subsection{Input data selection}

Pattern recognition and identification of highly nonlinear behaviors between variables by a neural network training algorithm is highly dependent on the appropriate selection of the training dataset. To achieve the successful mapping of the nonlinear behavior, the training dataset should be true representative of the nonlinear system. In this study, the available synchronized data of 13-month data were divided into two sets (i.e. training and testing). The total number of the time series of pore-water pressure and rainfall data consists of 4000 values. The data from Dec 1998 to July 1999 (3000) about 75\% of the data were used in training and from July 1999 to November 1999 (1000) about 25\% data (1000) for testing the model.

\subsection{Data normalization}

Preprocessing of the data in terms of scaling the complete data set into a specified range facilitate the network to converge easily. This ensures fast processing, increase the convergence rate during training and facilitates the network to minimize error during testing stage [16]. Since the pore-water pressure data contains negative pore-water pressure values as well as positive values. Therefore, the complete datasets of time series of pore-water pressure and rainfall data was normalized by transforming the data to the range of $(-1$ to 1) using the following equation

$$
X_{n}=2 \times \frac{\left(z_{n}-z_{\text {min }}\right)}{\left(z_{\text {max }}-z_{\text {min }}\right)}-1
$$

where, $X_{n}$ is the normalized data series, $z_{n}$ represents the real data series and $z_{\min }$, $z_{\max }$ are the minimum and the maximum value of the real data series respectively, $1 \leq n \leq N$ and $N$ is the number of data. 


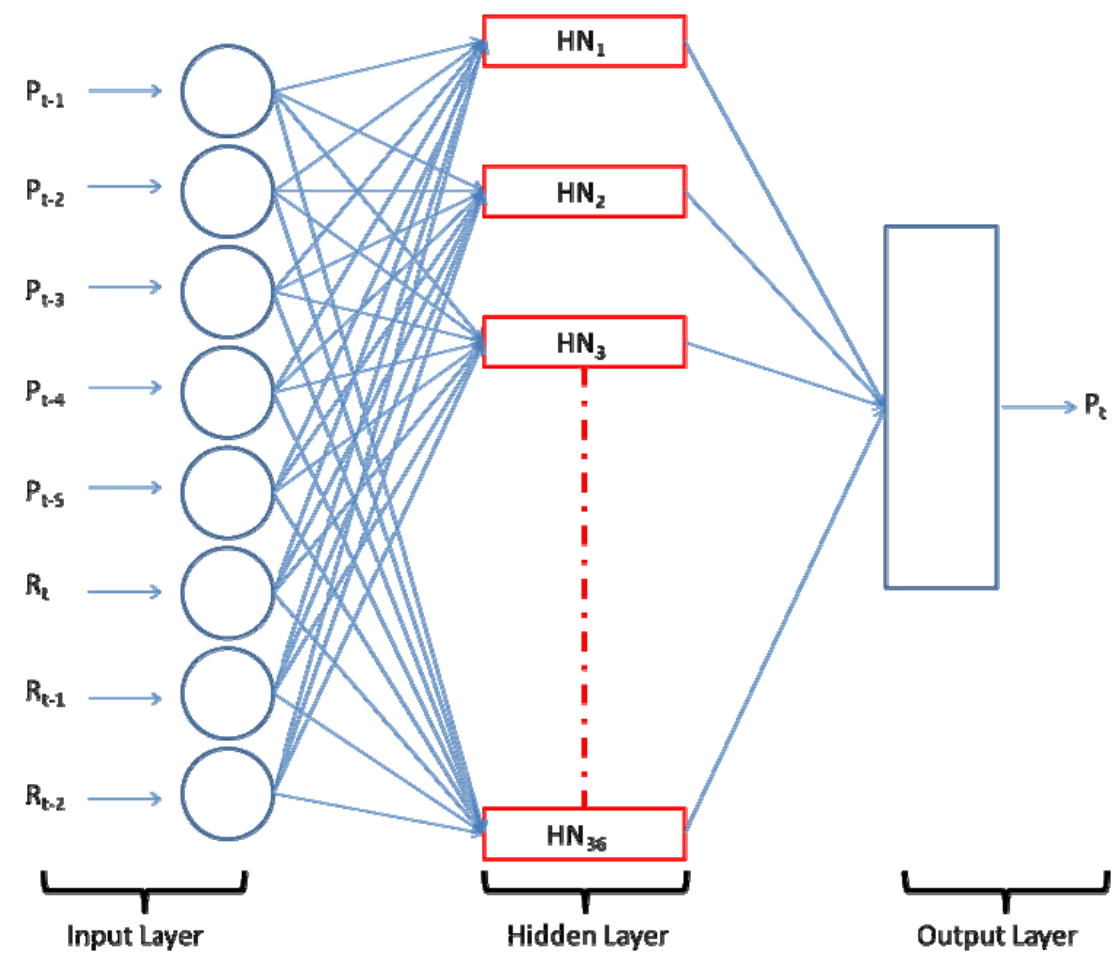

Figure 1: Schematic representation of RBF-TPS model architecture.

\subsection{Performance evaluation criteria}

Root mean square error (RMSE) is commonly used statistical measures to evaluate prediction accuracy in hydrological modeling. An RMSE value close to zero indicates good performance of the model. In this study, RMSE and coefficient of determination $\left(\mathrm{R}^{2}\right)$ were used to evaluate the performance of the predictions made by the model. Coefficient of determination indicates the closeness of the predicted values to the observed values and $\mathrm{R}^{2}$ values close to one indicates a good agreement between the observed and predicted data. The equation of RMSE is shown in the following equation;

$$
\operatorname{RMSE}=\left[\frac{1}{N} \sum_{n=1}^{N}\left(z_{n}-y_{n}\right)^{2}\right]^{1 / 2}
$$

where, $z_{n}$ and $y_{n}$ are the observed and predicted values of pore-water pressure, respectively, $N$ is the number of observations for which the error has been computed. 


\section{Results and discussion}

Radial basis function neural network model using Thin Plate Spline basis function was established with optimal network configuration of 8-36-1 (inputhidden-output neurons). The RBF-TPS model was able to follow the nonlinear behavior of soil pore-water pressure variations in response to rainfall patterns. The model was evaluated using commonly used statistical measures RMSE and coefficient of determination to examine the prediction accuracy between the observed and predicted values. The summary of performance statistics of the developed model during training and testing stages is shown in Table 1. The summary statistics shows that the RBF-TPS model produced RMSE $=0.8922$ and $\mathrm{R}^{2}=0.9915$ during training stage. This indicates the learning strength of the model during the network training process. The RBF-TPS model was trained very well and produces very small RMSE error. Coefficient of determination close to one suggests that the trained pore-water pressure data are very close to the observed data.

Table 1: Performance statistics of RBF-TPS model during training and testing.

\begin{tabular}{|l|c|c|}
\hline Performance criteria & Training & Testing \\
\hline RMSE & 0.8922 & 1.4098 \\
\hline $\mathrm{R}^{2}$ & 0.9915 & 0.9781 \\
\hline
\end{tabular}

However, it was observed that the RBF-TPS model produced slightly higher RMSE error $(\mathrm{RMSE}=1.4098)$ and lower coefficient of determination $\left(\mathrm{R}^{2}=\right.$ 0.9781) during testing stage as compared to the training. This difference in performance can be attributed to the difference of input and target data used during training and testing. Since during the training stage targets are presented to the network along with the input data to minimize the network error. Alternatively, only the input data is presented to the network during testing stage.

Time series of observed pore-water pressure data was compared with the predicted data during training stage, which indicates that the model has adequately captured the nonlinear pattern of the data (Figure 2). The predicted data track the exact behavior of observed values. It was observed that all the predicted data overlapped the observed data with an exception of very few values at the peaks. This difference between the observed and predicted values at the extreme values may be overcome by the use of clustering techniques. However, in this study these exceptions amongst a large dataset of 3000 values were ignored. A similar pattern was observed during the testing stage of the RBF-TPS model (Figure 3). The RBF-TPS model successfully predicted the observed data of pore-water pressure during the testing stage showing an excellent 
generalization ability of the developed model. However, very few data at the extreme values have slight difference between the observed and predicted data which was also observed during the training stage. Considering the highly nonlinear and complex behavior of pore-water pressure data, overfitting problem was not observed during any stage of model development.

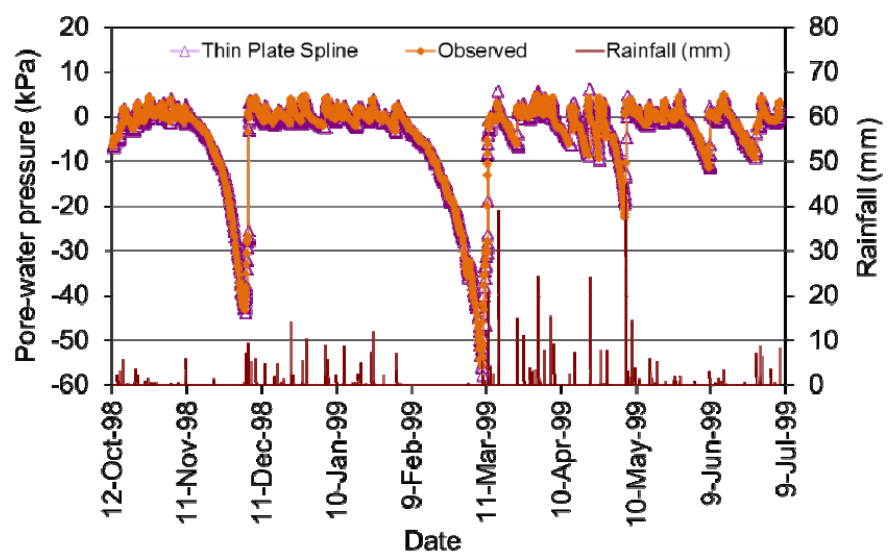

Figure 2: Comparison between the observed and predicted time series of pore-water pressure data during training the RBF-TPS model.

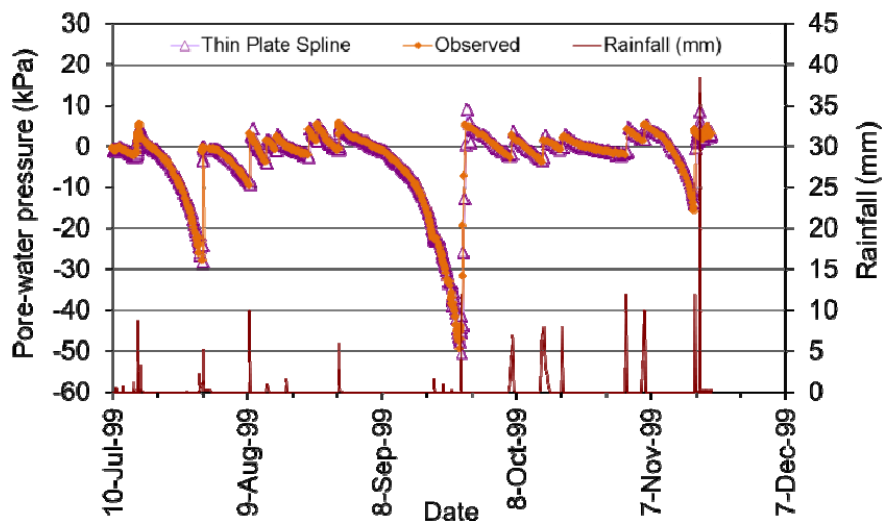

Figure 3: Comparison between the observed and predicted time series of pore-water pressure data during testing the RBF-TPS model.

A comparison between the predicted and observed pore-water pressure data with the line of perfect agreement during training and testing stages is shown in Figures 4 and 5 respectively. It is apparent from the plots that the predicted and observed pore-water pressure values showed a good agreement with the line of perfect agreement and produced coefficient of determination close to one $\left(\mathrm{R}^{2}=\right.$ 0.9915 (training) and $\mathrm{R}^{2}=0.9781$ (testing)). The difference between the 
observed and predicted values at the extreme positions particularly at the peak values which was observed in Figures 2 and 3, were appeared as outliers in the training and testing plots (Figures 4 and 5). However, disregarding the remarkable outliers, the synchronization between the observed and predicted data with line of perfect agreement recommends the suitability of the selected basis function for the prediction of pore-water pressure.

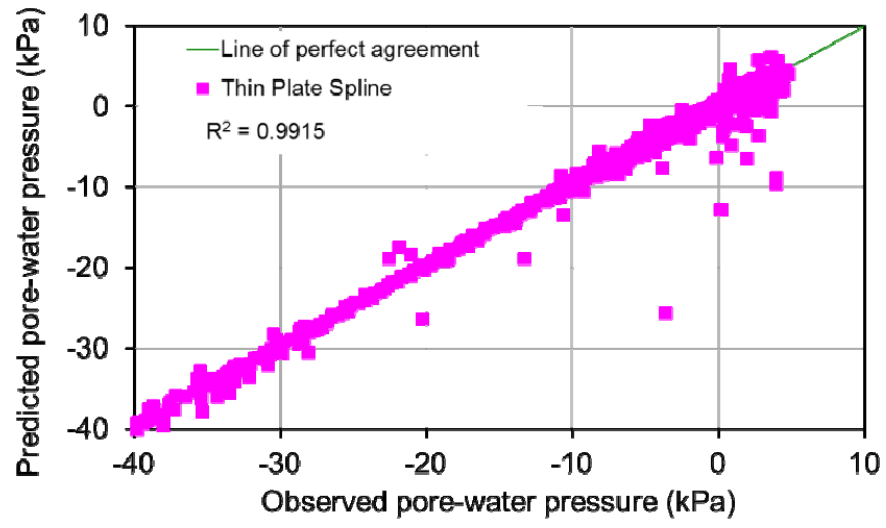

Figure 4: Comparison between the observed and predicted data with line of perfect agreement (training stage).

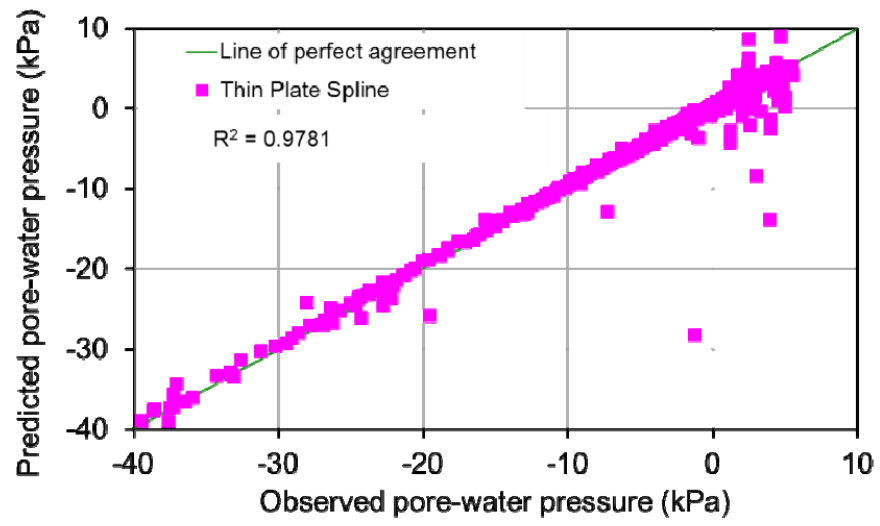

Figure 5: Comparison between the observed and predicted data with line of perfect agreement (testing stage).

Obviously, all the results recommend the suitability of Thin Plate Spline basis function neural network for prediction of soil pore-water pressure variations in response to the rainfall. The results obtained in this study are comparable with the previous studies [1-3] conducted by using Gaussian function and multilayer 
perceptron training algorithms. The results suggests that Thin Plate Spline basis function neural network can be used as an alternative to the Gaussian basis function and MLP training algorithms for prediction of variations of pore-water pressures. Disregarding to the tedious exercise involved in trial and error method for selection of appropriate number of basis functions. The architecture 8-36-1 with spread value of 1.8 was found appropriate to achieve high performance RBF-TPS prediction model for pore-water pressure variations.

\section{Conclusions}

A Radial Basis Function neural network model using Thin Plate Spline basis function has been developed to predict variations of a time series of pore-water pressure in response to rainfall in a slope. An appropriate network configuration identified as 8-36-1 with spread 1.8 was capable for mapping the complex behavior of pore-water pressure pattern responses to rainfall. The study indicated that RBF-TPS basis function is suitable for applications to solve complex problems and complications involved in prediction of nonlinear behavior between the variables such as pore-water pressure and rainfall. Furthermore, RBF-TPS model is competitive with the other modeling techniques such as Gaussian basis function, MLP training algorithms etc. for prediction of porewater pressure.

Although, the RBF-TPS model lacks the ability to provide a functional relationship between the variables unlike a physical based mathematical model, still it is advantageous as it can be developed with limited number of parameters. The results also showed a good agreement between predicted and observed data of pore-water pressure variations.

\section{Acknowledgement}

The authors gratefully acknowledge the facilities provided by the Universiti Teknologi PETRONAS to conduct this study.

\section{References}

[1] Mustafa M. R., Rezaur R. B., Rahardjo H., \& Isa M. H., Prediction of pore-water pressure using radial basis function neural network. Engineering Geology, 2012. 135-136: pp. 40-47.

[2] Mustafa M. R., Rezaur R. B., Isa M. H., Saiedi S., \& Rahardjo H., Effect of Antecedent Conditions on Prediction of Pore-Water Pressure Using Artificial Neural Networks. Modern Applied Science, 2012. 6(2): pp. 6-15.

[3] Mustafa M. R., Rezaur R. B., Saiedi, S., Rahardjo H. \& Isa M. H., Evaluation of MLP-ANN Training Algorithms for Modeling Soil PoreWater Pressure Responses to Rainfall. Journal of Hydrologic Engineering, 2013. 18(1): pp. 50-57. 
[4] Flood, I. and Kartam, N., Neural network in civil engineering-I: Principles and understanding. Journal of Computing in Civil Engineering, ASCE, 1994. 8(2): pp. 131-148.

[5] Mustafa, M. R., Isa M. H., \& Rezaur R. B., Artificial Neural Networks Modeling in Water Resources Engineering: Infrastructure and Applications International Journal of Social and Human Sciences, 2012. 6: pp. 240-248.

[6] Mustafa, M. R., Isa M. H., \& Rezaur R. B., Artificial Neural Networks Modeling in Water Resources Engineering: Infrastructure and Applications International. World Academy of Science, Engineering and Technology, 2012. 62: pp. 341-349.

[7] Mustafa M. R., Rezaur R. B., Saiedi S., \& Isa M. H., River Suspended Sediment Prediction Using Various Multilayer Perceptron Neural Network Training Algorithms-A Case Study in Malaysia. Water Resources Management, 2012. 26(7): pp. 1879-1897.

[8] Mustafa, M. R., Isa M. H., \& Rezaur R. B., A comparison of artificial neural networks for prediction of suspended sediment discharge in river-a case study in Malaysia. World Academy of Science, Engineering and Technology, 2011. 81: pp. 372-376.

[9] Mustafa, M. R. U., Isa M. H., and Rezaur R. B., Prediction of river suspended sediment load using radial basis function neural network-a case study in Malaysia. 2011.

[10] Han, H.-G., Chen Q.-1., and Qiao J.-F., An efficient self-organizing RBF neural network for water quality prediction. Neural Networks, 2011. 24(7): pp. 717-725.

[11] Tan X.-h., Bi W.-h., Hou X.-l., \& Wang W., Reliability analysis using radial basis function networks and support vector machines. Computers and Geotechnics, 2011. 38(2): pp. 178-186.

[12] Rahardjo, H., Leong E. C., \& Rezaur R. B., Effect of antecedent rainfall on pore-water pressure distribution characteristics in residual soil slopes under tropical rainfall. Hydrological Processes, 2008. 22(4): pp. 506-523.

[13] Meteorological-Service-Singapore, Summary of Observations (Annual Publication), Singapore, 1997.

[14] Tou, J.T. \& Gonzalez R.C., Pattern Recognition Principles. AddisonWesley, Reading, MA, 1974, 1974.

[15] Bishop, C.M., Neural Networks for Pattern Recognition. Oxford University Press, 1995: p. 482.

[16] Rojas, R., Neural Networks: A Systematic Introduction. Springer Verlag, Berlin, 1996: pp. 151-184. 